\title{
Autofluorescence of metastatic choroidal tumor
}

\author{
Tomoka Ishida - Kyoko Ohno-Matsui · Yuh Kaneko • \\ Hideaki Tobita $\cdot$ Kengo Hayashi $\cdot$ Noriaki Shimada $\cdot$ \\ Manabu Mochizuki
}

Received: 5 February 2008/Accepted: 19 May 2008/Published online: 5 June 2008

(C) The Author(s) 2008

\section{Introduction}

Metastatic choroidal carcinomas are the most common intraocular neoplasms [1]; diagnosis is made by ophthalmoscopic, fluorescein angiographic (FA), and indocyanine green angiographic (IA) findings together with a history of cancer especially of the breasts and lungs. However, the general health of these patients is often poor, and it is difficult to perform these invasive examinations. Fundus autofluorescence $(\mathrm{AF})$ is a noninvasive imaging technique that can detect lipofuscin in the retinal pigmented epithelial (RPE) cells [2]. A continuous AF pattern is an indication of normal metabolic activity of the RPE cells, and the loss of AF indicates that the photoreceptors or RPE cells are dysfunctional. Because FA and IA are generally not recommended in such ill patients, we questioned whether the AF pattern can be used to diagnose and follow the course of carcinomas after treatment. A Medline search did not extract any articles describing the AF findings in eyes with choroidal carcinomas.

The authors do not have financial interest in any products/drugs discussed in this article.

T. Ishida $\cdot$ K. Ohno-Matsui $(\bowtie) \cdot$ Y. Kaneko .

H. Tobita $\cdot$ K. Hayashi $\cdot$ N. Shimada $\cdot$ M. Mochizuki

Department of Ophthalmology and Visual Science,

Tokyo Medical and Dental University, 1-5-45 Yushima,

Bunkyo-ku, Tokyo 113, Japan

e-mail: k.ohno.oph@tmd.ac.jp
A retrospective review of patients with a choroidal carcinoma in our hospital extracted two patients who had undergone FA, IA, and AF. Thus, the purpose of this study was to determine the AF characteristics in these two eyes with a choroidal carcinoma.

\section{Case reports}

Case 1

A 35-year-old woman was examined to determine the cause of the vision decrease in her right eye. She had been diagnosed with breast cancer 1 year earlier and had undergone mastectomy and chemotherapy for metastasis to the lymph nodes, bones, lungs, and brain. Her best-corrected visual acuities (BCVA) were $0.7 \mathrm{OD}$ and 1.2 OS. A dome-shaped choroidal tumor with a yellowish depigmented area was seen ophthalmoscopically in the right eye (Fig. 1). FA showed granular hyperfluorescence along the margins of the tumor, and the depigmented area was hyperfluorescent in the late phase. An optical coherence tomographic (OCT) scan across the nasal margin of the tumor showed a dome-shaped elevation of the retina that was attached to the RPE-choriocapillaris complex, and a low-reflectance area corresponding to a retinal detachment (RD). IA revealed blocked fluorescence in the early phase and diffuse hyperfluorescence in the late phase. 


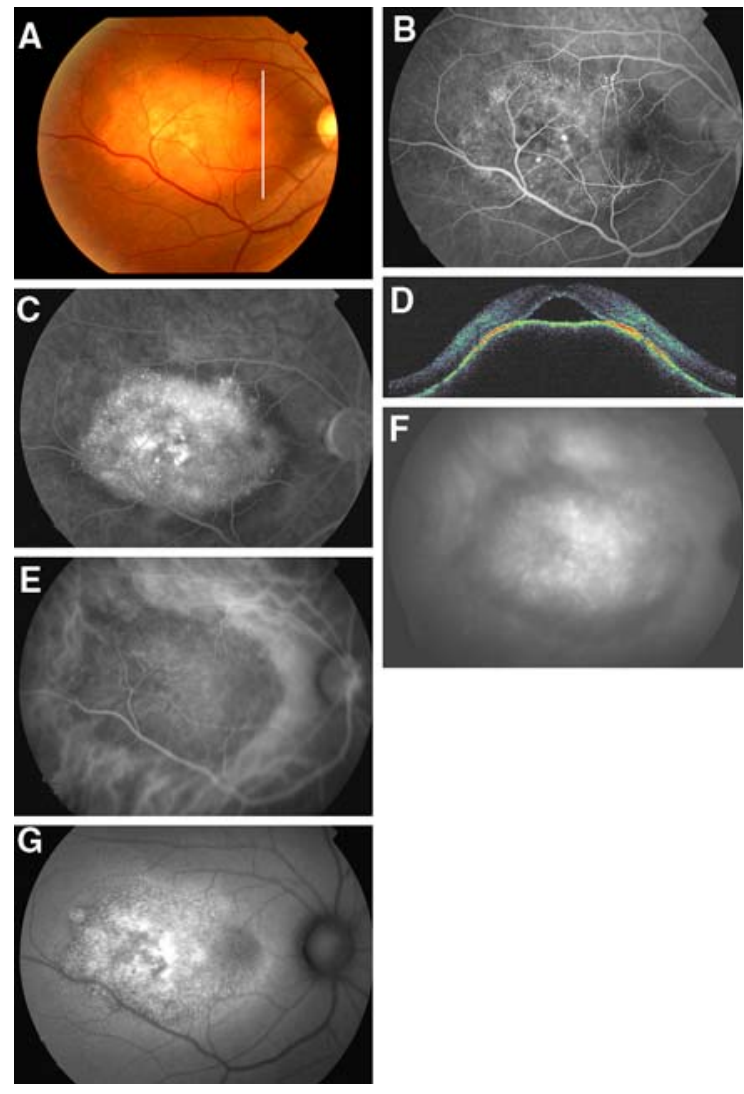

Fig. 1 Ophthalmic findings in case 1. (a) Fundus photograph of right eye showing a dome-shaped choroidal mass in the posterior fundus. Yellowish areas can be seen on the tumor. (b, c) Fluorescein angiograms showing granular hyperfluorescence along the margin of the tumor and hyperfluorescence of the yellowish area in (a). (d) An optical coherence tomographic image from a scan along the white line in (a). The image shows a dome-like elevation of the retina and RPE-choriocapillaris and RD overlying the tumor. (e, f) Indocyanine green angiograms showing blocked fluorescence in the early phase (e) and diffuse hyperfluorescence in the late phase (f). (g) Autofluorescence image, showing granular hypofluorescent dots along the margin of the tumor and hypofluorescence corresponding to the yellowish patch on the tumor

Autofluorescence examination with a fundus camera (TRC 50DX, Topcon, Japan) showed granular hypofluorescent dots along the margin of the tumor. This would suggest that the RPE cells overlying the tumor and along the margins of the tumor were damaged. Because her prognosis was extremely poor, irradiation was not performed.

One month later, her BCVA dropped to 0.04 and a massive hemorrhage was detected (Fig. 2). FA and AF showed a complete absence of fluorescence in most areas of the tumor. IA revealed a complete absence of the choroidal vasculature in most areas of the tumor, while large choroidal vessels remained only in the nasal area. In the nasal area, FA showed granular hyperfluorescence, and AF showed a predominant hypofluorescence (Fig. 2c). She died 5 days after the last examination.

\section{Case 2}

A 72-year-old woman was referred for metamorphopsia when using her left eye. She did not report having breast cancer or other malignancies. Initially, her BCVA was 1.2 OU. The left fundus showed a dome-shaped, creamy choroidal tumor superior to the macula (Fig. 3). FA showed granular hyperfluorescence along the margins of the tumor, and OCT showed a dome-shaped elevation of the retina attached to the RPE-choriocapillaris complex and a surrounding RD. AF was not performed because the equipment was not available.

From these findings, a metastatic choroidal tumor was suspected, and a systemic examination detected breast cancer with metastasis to the brain and lymph nodes. She underwent mastectomy, chemotherapy, and radiation to the left fundus and brain. Six months later, the choroidal mass was flat and yellowish patches appeared on the tumor ophthalmoscopically (Fig. 4). FA showed hyperfluorescence of the yellowish patches and hypofluorescence of the remaining tumor. AF showed that the yellowish patches were hypofluorescent and the remaining areas were hyperfluorescent. OCT showed a marked decrease in the thickness of the tumor and an absence of subretinal fluid.

\section{Comments}

The AF pattern in our two cases was approximately the reverse of the FA pattern. Thus, the granular hyperfluorescent dots seen by FA along the margins of the tumor were seen as hypofluorescent dots by AF. The hyperfluorescence of the yellowish pigments observed on the tumor by FA were hypofluorescent by AF. These fluorescent signals most likely indicate that the RPE cells around and overlying the tumor were damaged. 
Fig. 2 One month after the initial examination in case 1. (a) Right fundus shows massive hemorrhage overlying the tumor. (b, c) Fluorescein angiograms showing an absence of fluorescence in the temporal area of the tumor. Granular hyperfluorescence remains along the nasal margin of the tumor. (d)

Autofluorescence images showing an absence of fluorescence in the temporal area of the tumor. A mixture of hyper- and hypofluorescent dots are observed in the nasal area. $(\mathbf{e}, \mathbf{f})$ Indocyanine green angiograms showing a loss of the entire choroidal vasculature in the temporal area of the tumor from the early (e) to the late (f) angiograms. Only a small number of large choroidal vessels remain in the nasal area
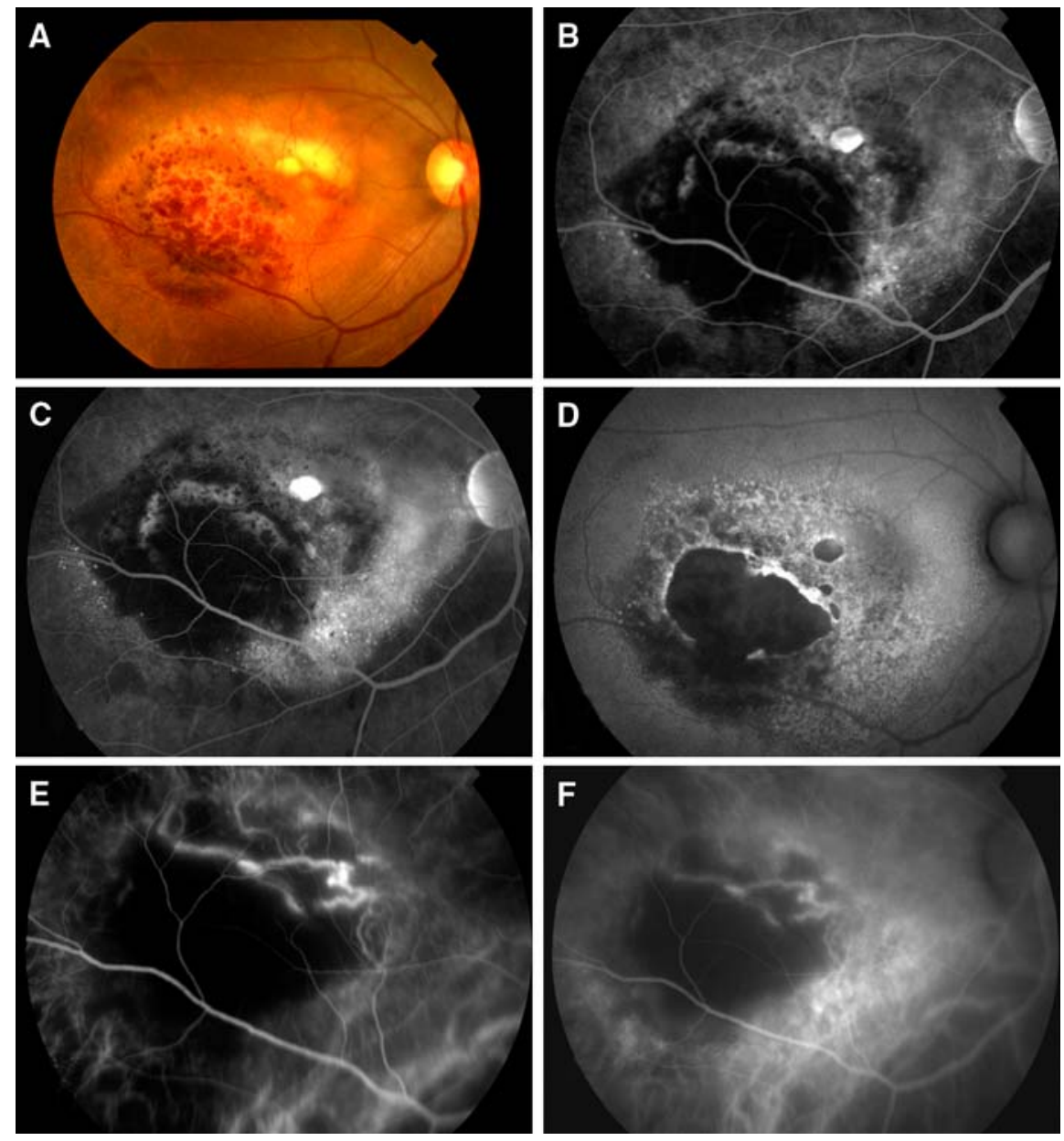

In case 1, the tumor became necrotic after chemotherapy, and IA suggested a necrosis of the entire choroidal vasculature, the RPE, and photoreceptors. This would explain the absence of fluorescence by both FA and $\mathrm{AF}$ in this area. However, the nasal area still showed hyperfluorescent dots by FA and hypofluorescent dots by AF, suggesting that the tumor was still perfused in this area but that the RPE cells did not contain lipofuscin.

After radiation in case 2, the AF pattern was the reverse of the FA pattern; yellowish patches were hyperfluorescent by FA and hypofluorescent by AF, reflecting a dysfunction of the RPE over the tumor. However, the remaining areas of the regressed tumor were intensely hyperfluorescent by AF and hypofluorescent by AF.

An increase in the AF signal has been reported in other intraocular tumors; an intense AF signal was reported in eyes with a choroidal melanoma, and a histopathological study of the enucleated eye revealed hyperautofluorescent deposits within the RPE and macrophages overlying the melanoma $[3,4]$.

Cytoplasmic lipofuscin granules have been demonstrated in breast tissue obtained before chemotherapy and radiation [5]. Light microscopic examinations of the excised mammary tissues from our case did not detect any autofluorescent lipofuscin pigment in the tissue. Unfortunately, the excised mammary tissues were already formalin-fixed, and electron microscopy was not possible.

After radiation, the released lipofuscin from necrotic tumor cells might have been taken up by the RPE cells and macrophages overlying the tumor, and this accumulated lipofuscin might have been manifested as the intense AF signal. 
Fig. 3 Ophthalmic findings in case 2. (a) Left fundus shows a creamy choroidal tumor superior to the macula. (b, c) Fluorescein angiograms showing granular hyperfluorescence along the margin of the tumor; late phase (c) shows diffuse hyperfluorescence. (d) Optical coherence tomographic scan along the line in (a), showing a domelike elevation of the retina and RPE-choriocapillaris complex and surrounding retinal detachment
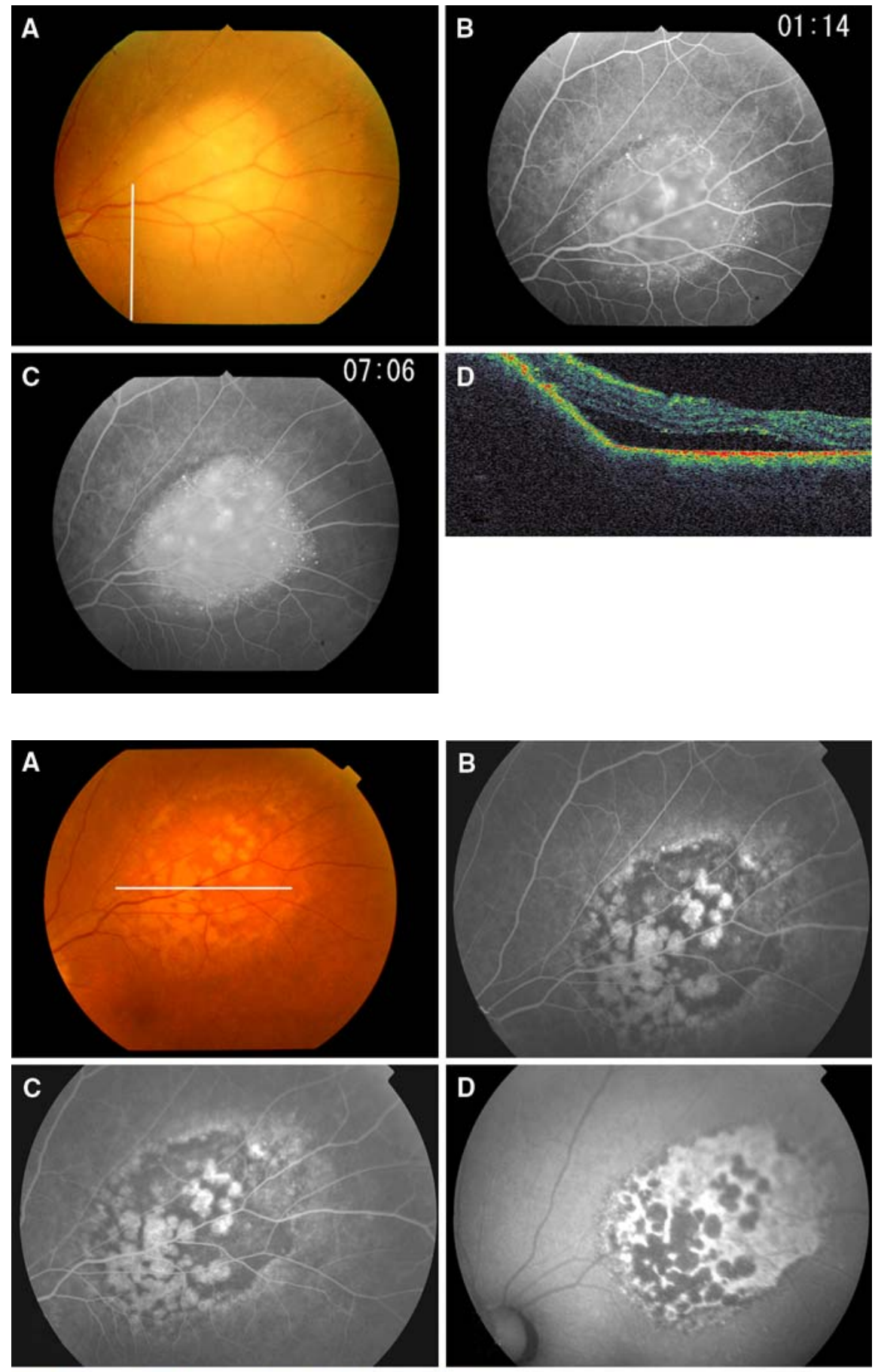

E
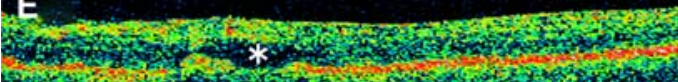

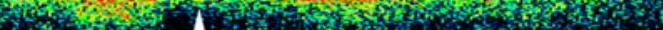

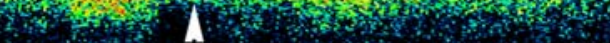


Although further studies are necessary, noninvasive AF might be clinically useful for diagnosis as well as the choice of therapy type.

Acknowledgments The authors thank Prof. Duco Hamasaki for his critical discussion and revision of the final manuscript. Supported in part by research grant 19390441 and 19659445 from the Japan Society for the Promotion of Science, Tokyo, Japan.

Open Access This article is distributed under the terms of the Creative Commons Attribution Noncommercial License which permits any noncommercial use, distribution, and reproduction in any medium, provided the original author(s) and source are credited.

\section{References}

1. Shields JA, Shields CL (1992) Intraocular tumors: a text and atlas. WB Saunders, Philadelphia, pp 207-238
2. Sparrow JR (2007) Lipofuscin of the retinal pigment epithelium. In: Holz FG, Schmitz-Valckenbeg S, Spaide RF, Bird AC (eds) Atlas of fundus autofluorescence imaging. Springer, Heidelberg, pp 1-16

3. Lavinsky D, Belfort RN, Navajas E et al (2007) Fundus autofluorescence of choroidal nevus and melanoma. $\mathrm{Br} \mathrm{J}$ Ophthalmol 91(10):1299-1302

4. Font RL, Zimmerman LE, Armaly MF (1974) The nature of the orange pigment over a choroidal melanoma. Arch Ophthalmol 91(5):359-362

5. Shin SJ, Kanomata N, Rosen PP (2000) Mammary carcinoma with prominent cytoplasmic lipofuscin granules mimicking melanocytic differentiation. Histopathology 37(5):456-459 